\title{
\begin{tabular}{l|l} 
Mibraries & DSpace@MIT
\end{tabular}
}

\author{
MIT Open Access Articles
}

Unidirectional propagation of magnetostatic surface spin waves at a magnetic film surface

The MIT Faculty has made this article openly available. Please share how this access benefits you. Your story matters.

Citation: Wong, Kin L., Lei Bi, Mingqiang Bao, Qiye Wen, Jean Pierre Chatelon, Yen-Ting Lin, C. A. Ross, Huaiwu Zhang, and Kang L. Wang. "Unidirectional Propagation of Magnetostatic Surface Spin Waves at a Magnetic Film Surface." Applied Physics Letters 105, no. 23 (December 8, 2014): 232403. ( 2014 AIP Publishing LLC

As Published: http://dx.doi.org/10.1063/1.4903742

Publisher: American Institute of Physics (AIP)

Persistent URL: http://hdl.handle.net/1721.1/102185

Version: Final published version: final published article, as it appeared in a journal, conference proceedings, or other formally published context

Terms of Use: Article is made available in accordance with the publisher's policy and may be subject to US copyright law. Please refer to the publisher's site for terms of use. 


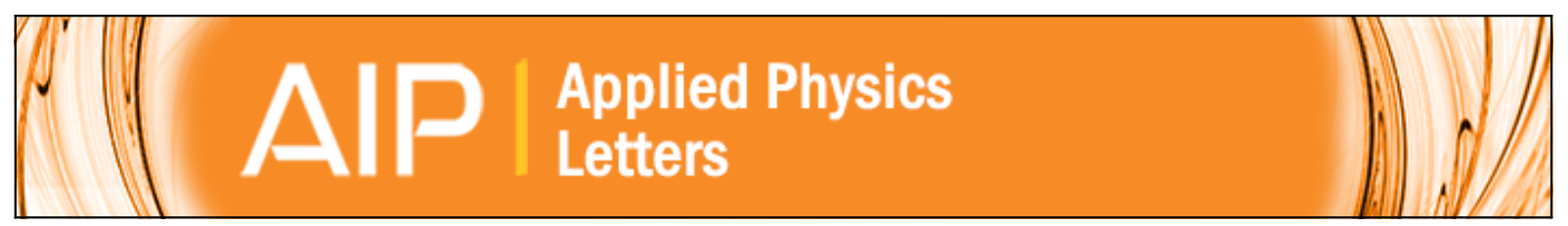

\section{Unidirectional propagation of magnetostatic surface spin waves at a magnetic film}

\section{surface}

Kin L. Wong, Lei Bi, Mingqiang Bao, Qiye Wen, Jean Pierre Chatelon, Yen-Ting Lin, C. A. Ross, Huaiwu Zhang, and Kang L. Wang

Citation: Applied Physics Letters 105, 232403 (2014); doi: 10.1063/1.4903742

View online: http://dx.doi.org/10.1063/1.4903742

View Table of Contents: http://scitation.aip.org/content/aip/journal/apl/105/23?ver=pdfcov

Published by the AIP Publishing

\section{Articles you may be interested in}

Magnetostatic surface wave propagation in a one-dimensional magnonic crystal with broken translational symmetry

Appl. Phys. Lett. 101, 242408 (2012); 10.1063/1.4771126

Hot spin-wave resonators and scatterers

J. Appl. Phys. 112, 013902 (2012); 10.1063/1.4730927

Mode-conversion enhancement of guided optical waves by magnetostatic surface waves propagating collinearly in obliquely magnetized bismuth-doped yttrium-iron-garnet film waveguide

J. Appl. Phys. 100, 123104 (2006); 10.1063/1.2399883

Wave front reversal of nonreciprocal surface dipolar spin waves

J. Appl. Phys. 99, 08P513 (2006); 10.1063/1.2172184

Parametric and modulation instabilities of magnetostatic surface spin waves in ferromagnetic films

J. Appl. Phys. 81, 1341 (1997); 10.1063/1.363869

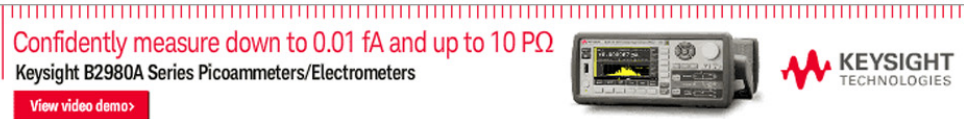




\title{
Unidirectional propagation of magnetostatic surface spin waves at a magnetic film surface
}

\author{
Kin L. Wong, ${ }^{1}$ Lei Bi, ${ }^{2,3}$ Mingqiang Bao, ${ }^{1, a)}$ Qiye Wen, ${ }^{3}$ Jean Pierre Chatelon, ${ }^{4}$ \\ Yen-Ting Lin, ${ }^{1}$ C. A. Ross, ${ }^{2, a)}$ Huaiwu Zhang, ${ }^{3}$ and Kang L. Wang ${ }^{1}$ \\ ${ }^{1}$ Department of Electrical Engineering, University of California, Los Angeles, Los Angeles, California 90095 , \\ USA \\ ${ }^{2}$ Department of Materials Science and Engineering, Massachusetts Institute of Technology, Cambridge, \\ Massachusetts 02139, USA \\ ${ }^{3}$ State Key Laboratory of Electronic Thin Films and Integrated Devices, University of Electronic Science \\ and Technology of China, Chengdu 610054, China \\ ${ }^{4}$ Univerisité de Saint-Etienne, Université de Lyon, LT2C, 25 rue du Docteur Rémy Annino, 42000 \\ Saint-Etienne, France
}

(Received 21 September 2014; accepted 25 November 2014; published online 8 December 2014)

\begin{abstract}
An analytical expression for the amplitudes of magnetostatic surface spin waves (MSSWs) propagating in opposite directions at a magnetic film surface is presented. This shows that for a given magnetic field $H$, it is forbidden for an independent MSSW to propagate along the direction of $-\vec{H} \times \vec{n}$, where $\vec{n}$ is the surface normal. This unidirectional propagation property is confirmed by experiments with both permalloy and yttrium iron garnet films of different film thicknesses, and has implications in the design of spin-wave devices such as isolators and spin-wave diodes. (C) 2014 AIP Publishing LLC. [http://dx.doi.org/10.1063/1.4903742]
\end{abstract}

Spin waves are a manifestation of the high-frequency fundamental dynamics of ferromagnets and ferrites, and have been a hot topic for more than five decades. ${ }^{1-11}$ The propagation of magnetostatic surface spin waves (MSSWs) is known to be nonreciprocal. ${ }^{12-16}$ However, the wave amplitude relationship of spin waves propagating in different directions is still not established quantitatively, even for the simplest case of determining the relationship between the amplitudes of two waves excited by a single source and propagating in opposite directions at the same surface. Interest in this topic has been growing recently since MSSWs have been considered as one of the potential candidates for future information processing systems. ${ }^{17-19}$ Recent progress in nanofabrication also allows new spin wave device functionalities to be explored. ${ }^{7-11}$ In this work, we present the quantitative relationship between the amplitude of MSSWs propagating in opposite directions at the same film surface, and show that it is forbidden for an independent MSSW to propagate in the direction of $-\vec{H} \times \vec{n}$, where $\vec{H}$ is the effective magnetic field in the film and $\vec{n}$ is the surface normal. Further to the known nonreciprocal propagation, this paper will show that the MSSW propagation at the surface is intrinsically unidirectional: It has only one sign of wavevector, while the observed wavevector with opposite $\operatorname{sign}^{12-16}$ is due to leakage from the other surface of the film due to limited thickness, and the amplitude of those leaked waves will reduce exponentially as the film becomes thicker. This conclusion may lead to the realization of spin-wave isolators and spin-wave diodes.

Figure 1 shows a magnetic film with infinite lateral extent with a magnetic field $H$ applied in the film plane along the z-axis. In this work, only MSSWs are considered since it is easier to excite and propagate a single mode MSSW than a backward volume magnetostatic spin wave (BVMSW) ${ }^{2}$ and,

a)Electronic addresses: mingqiangbao@gmail.com and caross@mit.edu based on our experiments, the attenuation of a BVMSW in a metallic ferromagnetic film is orders higher than that of an MSSW. Without loss of generality, the MSSW is assumed to propagate along the $\mathrm{y}$-axis and $k_{z}=0 .{ }^{2,6}$ Therefore, waves propagating at the top film surface can be written as $A_{+y} e^{i\left(\omega t-\left|k_{y}\right| y\right)}$ for the wave propagating along the $+y$ direction, and $B_{-y} e^{i\left(\omega t+\left|k_{y}\right| y\right)}$ for the wave along the $-\mathrm{y}$ direction. Similarly, the waves at the bottom surface can be written as $C_{+y} e^{i\left(\omega t-\left|k_{y}\right| y\right)}$ and $D_{-y} e^{i\left(\omega t+\left|k_{y}\right| y\right)}$, respectively.

We follow Damon and Eshbach's approach ${ }^{2}$ and notation to obtain the simultaneous solution of Maxwell's equations and the equation of motion of the sample magnetization, with the boundary conditions of the continuity of the normal component of the $\vec{B}$ field and the tangential $\vec{H}$ field at the surfaces. The amplitude ratio of those waves can be subsequently obtained, i.e., the solution to Eq. (17) of Ref. 2 or

$$
\frac{A_{+y}}{C_{+y}}=\frac{D_{-y}}{B_{-y}}=\frac{1}{2}\left(e^{\left|k_{y}\right| s}+e^{-\left|k_{y}\right| s}\right)+\frac{1+|\nu|}{1+\kappa}\left[\frac{1}{2}\left(e^{\left|k_{y}\right| s}-e^{-\left|k_{y}\right| s}\right)\right],
$$

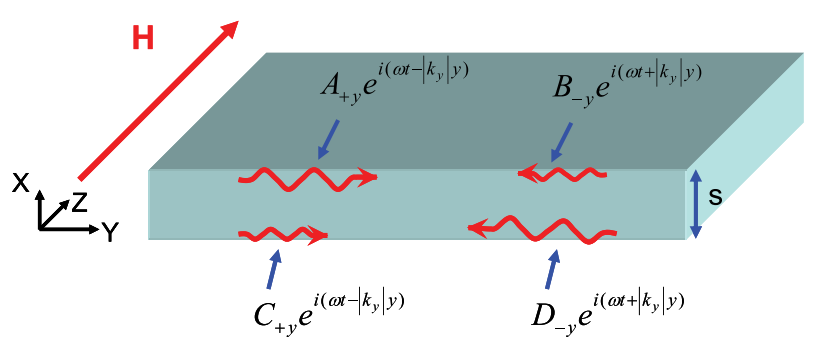

FIG. 1. Magnetic film and its coordinate orientation. The external magnetic field is in the film plane along the z-axis. $A_{+y}$ and $B_{-y}$ are the amplitudes of surface spin waves propagating in opposite directions at the top surface, while $C_{+y}$ and $D_{-y}$ are the amplitudes of surface spin waves propagating in opposite directions at the bottom surface. The film thickness is $s$. 
where $1+\kappa=\frac{\pi M_{0}}{\pi M_{0}+H /\left(1-e^{-2\left|k_{y}\right| s}\right.}, \nu=\frac{\omega / \gamma}{H+\pi M_{0}\left(1-e^{-2|k| s \mid s}\right)}, M_{O}$ is the saturation magnetization of the magnetic film, $s$ is the film thickness, $\gamma$ is the gyromagnetic ratio, and

$$
\omega=-\gamma\left(H^{2}+4 \pi M_{0} H+\left(2 \pi M_{0}\right)^{2}\left(1-e^{-2\left|k_{y}\right| s}\right)\right)^{1 / 2} .
$$

Since the system of Figure 1 is identical as $x \rightarrow-x$ and $y \rightarrow-y$, it is straightforward to write that $A_{+y}=D_{-y}$ and $B_{-y}=C_{+y}$ for the same $\left|k_{y}\right|$ value under the same excitation condition. Thus, we have

$$
\frac{A_{+y}}{B_{-y}}=\frac{1}{2}\left(e^{\left|k_{y}\right| s}+e^{-\left|k_{y}\right| s}\right)+\frac{1+|\nu|}{1+\kappa}\left[\frac{1}{2}\left(e^{\left|k_{y}\right| s}-e^{-\left|k_{y}\right| s}\right)\right] .
$$

Since $1+\kappa=\frac{\pi M_{0}}{\pi M_{0}+H /\left(1-e^{-2\left|k_{y}\right| s}\right)}, \nu=\frac{\omega / \gamma}{H+\pi M_{0}\left(1-e^{-2\left|k_{y}\right| s}\right)}$, and from Eq. (2), $\frac{1+|\nu|}{1+\kappa}$ can be easily simplified as $\frac{1+|\nu|}{1+\kappa}>\frac{1+\frac{\left(2 \pi M_{0}\right)\left(1-e^{\left.-2|k| k_{y} \mid s\right)}\right.}{H+\pi M_{0}\left(1-e^{-2\left|k_{y}\right| s}\right)}}{1+\kappa}$ $>3$, and Eq. (3) can consequently be simplified as $\frac{A_{+y}}{B_{-y}}>2 e^{\left|k_{y}\right| s}-e^{-\left|k_{y}\right| s}$. This clearly demonstrates that the amplitude of the wave propagating along the $+y$ direction is exponentially larger than that of the wave along the $-y$ direction at the top surface. For more details, the following three distinct situations are discussed:

Thick film: For a thick film ( $s \gg \lambda$, where $\lambda$ is the wavelength of the spin wave), as $\left|k_{y}\right| s \gg 1$, then Eq. (3) reduces to $A_{+y} / B_{-y} \gg 1$. This means $B_{-y}=0$ since the spin wave amplitude $A_{+y}$ cannot be infinitely large. This indicates that under this condition, the MSSW propagates only along the $+y$ direction at the top surface. If the system is symmetrical, at the bottom surface the wave can propagate only along the $-y$ direction. In summary, the MSSW propagates only along the direction of $\vec{H} \times \vec{n}$ at either the top surface or the bottom surface.

Intermediate film thickness; For a film whose thickness $s=\lambda$, Eq. (3) reduces to $\left(\frac{A_{+y}}{B_{-y}}\right)^{2}>10^{6}$, which demonstrates that the spin wave energy along the $+y$ direction is more than $10^{6}$ times larger than that along the $-y$ direction. This implies that even a thin film, for example, $100 \mathrm{~nm}$ thick, an excellent non-reciprocity of the signal transmission could be still achieved if the wavelength is close to or less than the film thickness.

Thin film: For a thin film where $s \ll \lambda$ and $\left|k_{y}\right| s \ll 1$, Eq. (3) can be simplified to

$$
\frac{A_{+y}}{B_{-y}}=1+\frac{1+|\nu|}{1+\kappa}\left(\left|k_{y}\right| s\right) .
$$

Equation (4) indicates the waves can propagate in both directions, but the amplitude of an MSSW along the $+y$ direction is larger than that along the $-y$ direction at the top surface.

Further examining the surface wave distribution, we find the waves are attenuated within a characteristic length $1 /\left|k_{y}\right|$ as the wave travels away from the surface of the film toward either the interior or exterior of the film. With the same approach as Ref. 2, the decay of the transverse magnetic potential in the $x$-direction is the same as Eq. (1), where $s$ is substituted for $x$. From the decay of the transverse magnetic potential in the $x$-direction, it is clear that the energy is localized at either the top or bottom surface, and there is only one wave propagating at the top surface, that is, along the $+y$ direction; and the wave propagating at bottom surface is only along the $-y$ direction. Thus, physically, $C_{+y}$ is the leaked signal of $A_{+y}$ and $B_{-y}$ is the leaked signal of $D_{-y}$ because the film is so thin that the film is unable to isolate MSSWs completely. As the film thickness $s$ increases, $B_{-y}$ and $C_{+y}$ will decrease exponentially. If there is no wave propagating along the $\vec{H} \times \vec{n}$ direction, an MSSW is unable to propagate along the $-\vec{H} \times \vec{n}$ direction at the film surfaces alone. In this sense, we conclude that under a magnetic field $H$, it is forbidden for an independent MSSW to propagate along the direction of $-\vec{H} \times \vec{n}$.

The property of unidirectional propagation of MSSWs at the film surface may lead to potential applications, for example, spin-wave diodes and isolators, and Eq. (3) will serve as the design reference. In addition, it is interesting to study how an MSSW interacts with an obstacle because the simple reflection of a MSSW at an obstacle is forbidden.

Finally, we note that exchange interaction had been ignored in the above analysis. However, the unidirectional propagation of MSSW is still valid even when exchange interaction is taken into consideration, and the effective magnetic field $H$ in the above equations now becomes $H+\frac{2 A}{M_{0}} k^{2},{ }^{20}$ where $A$ is the exchange stiffness constant of the magnetic film.

In order to verify Eq. (3) and the theoretical prediction of unidirectional propagation of MSSW, a series of prototype devices have been fabricated. The first one is a $20 \mathrm{~nm}$ thick polycrystalline permalloy $\left(\mathrm{Ni}_{80} \mathrm{Fe}_{20}\right)$ film deposited by sputtering onto thermally oxidized $\mathrm{Si}$ substrate. The prototype device is shown as the inset in Figure 2: two antennas with $4 \mu \mathrm{m}$ wide $200 \mathrm{~nm}$ thick gold wires are deposited by sputtering on top of the permalloy film, separated from the permalloy by a layer of $200 \mathrm{~nm} \mathrm{SiO}$. Figure 2 shows the amplitude in logarithmic scale $\mathrm{dBm}$ of spin waves propagating along the direction from Antenna 1 to Antenna 2 under a negative (along the $-z$ direction) and positive (along the $+z$ direction) magnetic field $H^{21}$ A linear plot at the frequency of $2.75 \mathrm{GHz}$ is shown in the top panel. This shows that the spin-wave amplitude at $H>0$ is larger than that at $H<0$ for the same wave-vector. At $2.75 \mathrm{GHz}$, the wave amplitude ratio of two outer peaks, one at $H \approx 100 \mathrm{Oe}$ and another at $H \approx-100 \mathrm{Oe}$, is approximately 1.2 , which is the same value as that predicted by Eq. (4) at the wavelength of $16 \mu \mathrm{m}$.

Strictly speaking, the top antenna structure breaks the symmetrical condition of $x \rightarrow-x$ that has been used to derive Eq. (3). However, our experimental data shows that the MSSW power detected by the metal line (antenna structure) is less than $-45 \mathrm{dBm}$ when $0 \mathrm{dBm}$ microwave has been used to excite MSSWs. Thus, the detected MSSW power is more than 30000 times smaller than the excitation power. This indicates a very weak coupling between the MSSW and antenna. Therefore, even though the top antenna exists, Eq. (3) is still valid as a good quantitative estimation formula because the top metal line has a very limited effect on MSSW propagation. Further evidence that the antenna is not important is that MSSWs can be excited and detected by a purely optical technique that eliminates the top metal antennas. For example, Ref. 14 used Brillouin light-scattering 


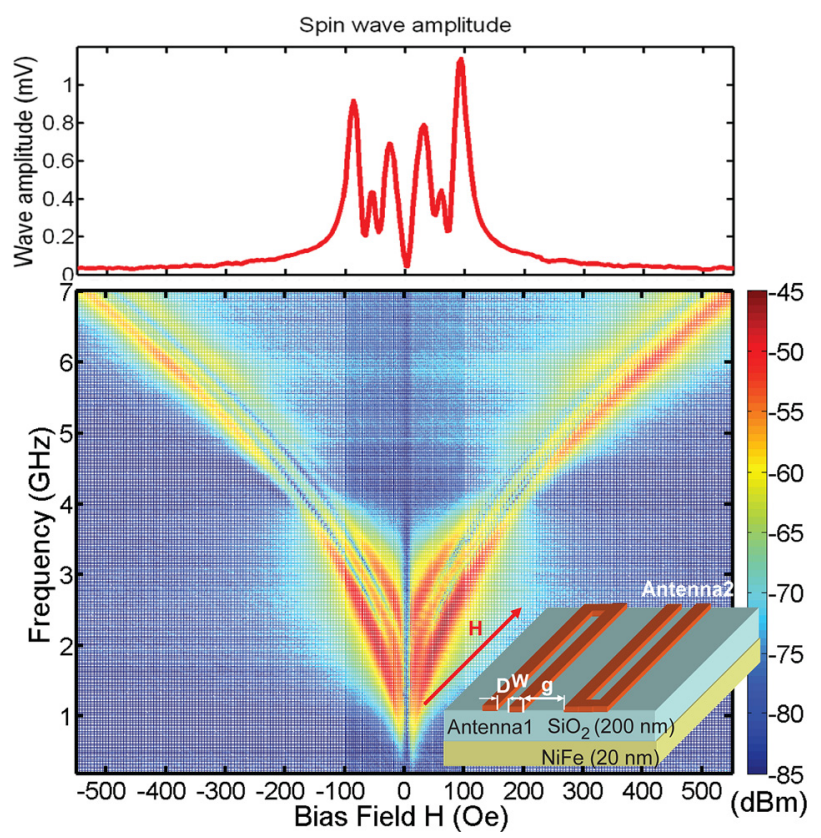

FIG. 2. The amplitude in logarithmic scale $\mathrm{dBm}$ of spin waves propagating from Antenna 1 to Antenna 2. The spin-wave excitation energy is $0 \mathrm{dBm}$. A linear plot at a frequency of $2.75 \mathrm{GHz}$ is shown in the top panel. The device structure is shown in the right inset: two antennas with dimensions of $D=W=4 \mu \mathrm{m}$. The gap $g$ between the two antennas is $8 \mu \mathrm{m}$. Three branches corresponding to different frequencies are evident at $H>0$ and $H<0$, respectively. These patterns occur because the antenna is at a node in the intensity when the wavelength of a spin wave is either $D+W$ or $(D+W) / 2$ which results in a zero output. In addition, the antenna becomes much less efficient for wavelengths below the gold-wire width $W$, which explains why only three branches were observed, instead of many branches.

spectroscopy to detect MSSWs. The conventional microwave antenna method and the optical method are therefore consistent.

Figure 3 shows the spin-wave amplitude ratios $A_{+y} / B_{-y}$ propagating in opposite directions at the top surfaces of a $20 \mathrm{~nm} \mathrm{NiFe}$ film, a $160 \mathrm{~nm}$ yttrium iron garnet (YIG) film, and a $2 \mu \mathrm{m}$ YIG film. The $160 \mathrm{~nm}$ YIG was made by pulsed laser deposition on a (111)-oriented gallium gadolinium garnet (GGG) substrate and is a single crystal with saturation magnetization close to bulk. The $2 \mu \mathrm{m}$ YIG film was made by liquid phase epitaxial growth on (111)-oriented GGG substrate. The data for the $20 \mathrm{~nm} \mathrm{NiFe}$ film fit well with Eq. (3); while for the $160 \mathrm{~nm}$ YIG films, the measured amplitude ratio is a little larger than that predicted by Eq. (3); and for $2 \mu \mathrm{m}$ YIG film, the measured amplitude ratio is much larger (almost 2 times) than that predicted by Eq. (3).

At the small signal level, the MSSW amplitude excited by the antennas varies linearly with the maximum microwave magnetic field at the film surface generated by the microwave current in the antenna. ${ }^{5}$ With our current asymmetric experimental setup, the MSSW signal excited by the top antennas is weaker at the bottom film surface than at the top surface because the bottom surface is farther away from the antennas resulting in smaller MSSW amplitude at the bottom surface. The solid lines show the modified results after compensation for the different magnetic fields generated by the antenna at two different locations: one located at the top magnetic film surface that is $200 \mathrm{~nm}$ underneath the $4 \mu \mathrm{m}$ gold wire, and another at the bottom surface, $200 \mathrm{~nm}$ plus the magnetic film

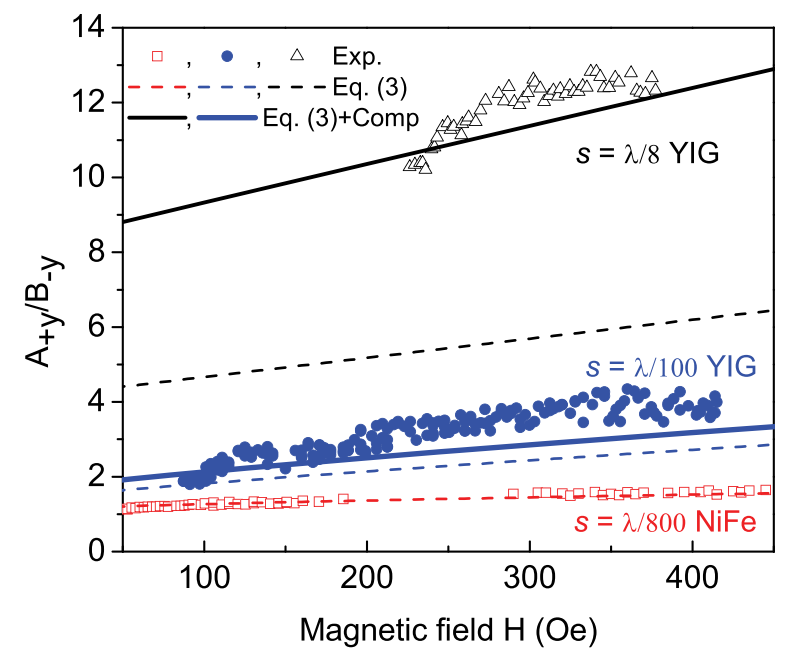

FIG. 3. Amplitude ratios of MSSWs propagating in opposite directions for three magnetic film thicknesses: $s=\lambda / 800=20 \mathrm{~nm}$ NiFe film, $s=\lambda / 100$ $=160 \mathrm{~nm}$ YIG film, and $s=\lambda / 8=2 \mu \mathrm{m}$ YIG film, where the wavelength $\lambda=16 \mu \mathrm{m}$ is used in the calculation. ${ }^{7,17}$ Scatter points are from experimental results; dashed lines are theoretical calculations from Eq. (3), where $4 \pi M_{0}=9.87 \mathrm{kG}$ for $\mathrm{NiFe}$ and $4 \pi M_{0}=1.78 \mathrm{kG}$ for YIG have been used in the calculation; and solid lines are Eq. (3) plus a compensation for different spin wave amplitudes excited by an antenna at different distances to the magnetic film surfaces. The test devices for YIG films are the same as the $\mathrm{NiFe}$ device in the inset of Figure 2 except the $\mathrm{NiFe}$ film on $\mathrm{SiO}_{2}$ layer is replaced by a YIG film on GGG single crystal substrate.

thickness $s$. After the distance compensation, the experimental data and the theory predictions now are in good agreement. This is additional evidence that MSSWs propagating along the $-y$ direction cannot be directly excited at the top surface by the antennas; however, they are excited at the bottom surface $\left(D_{-y}\right)$ first, then attenuated through the magnetic film, and finally detected by the top antenna $\left(B_{-y}\right)$.

The last situation we consider is spin-wave propagation at the surface of a thick magnetic substrate, a $254 \mu \mathrm{m}$ thick YIG crystal. The antennas for excitation and detection of spin waves are the same size as the antennas in Figure 2. For this thick YIG film, we were unable to obtain the spin-wave amplitude directly. Since the magnetic film is so thick that $|k| s \gg 1$, Eq. (2) can be simplified to $\omega=-\gamma\left(H+2 \pi M_{0}\right)$. This yields the result that all spin waves with different wave-vectors will degenerate into the same dispersion curve, and the spin wave frequency is linear in magnetic field $H$. Under this condition, we cannot detect clear spin wave signals with separated wave-vectors. We therefore measure the relative signal amplitude from Antenna 1 to Antenna 2 (S21) and the amplitude from Antenna 2 to Antenna 1 (S12) in $d B$, instead of the spin wave amplitude in $\mathrm{dBm}$. S21 and S12 are measured by a vector network analyzer (VNA) connected to Antenna 1 and Antenna 2. Figure 4 shows S21 in a logarithmic scale of $\mathrm{dB}$. The $\mathrm{S} 21$ results clearly show a narrow linear relation which fits the equation $\omega=-\gamma\left(H+2 \pi M_{0}\right)$. In contrast, such a feature is absent in the S12 data. If the field direction is reversed, the same linear relation appears in the S12 data while not in S21. Figure 4 therefore shows that for a thick film, there are spin waves that propagate from Antenna 1 to Antenna 2 at the top surface, while no spin waves propagate from Antenna 2 to Antenna 1. Therefore, $B_{-y}=0$ and $A_{+y} / B_{-y} \gg 1$, which is consistent with Eq. (3). 


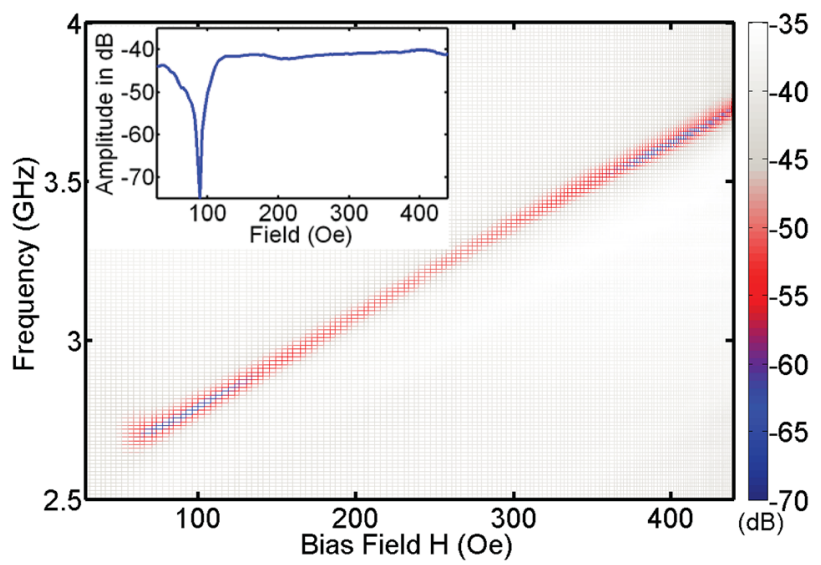

FIG. 4. The relative signal amplitude in logarithmic scale $d B$ from Antenna 1 to Antenna $2(\mathrm{~S} 21)$ on a $254 \mu \mathrm{m}$ thick YIG substrate. There is a straight line feature in the S21 data. There is no spin wave observed from Antenna 2 to Antenna 1. The amplitude at a frequency of $2.75 \mathrm{GHz}$ from the $\mathrm{S} 21$ data is shown in the inset. A sharp deep singular valley occurs at an $H$ field of 90 Oe and its line width is less than 1 Oe.

To conclude, we have derived a quantitative relationship connecting the amplitudes of MSSWs propagating in opposite directions at the same surface of a magnetic film, and shown that the propagation of MSSWs is intrinsically unidirectional and has only one sign of wavevector: for a thick film (the film thickness is larger than the wavelength of the MSSW), MSSWs are only allowed to propagate along the $\vec{H} \times \vec{n}$ direction, and to propagate in the opposite direction is forbidden; while a thin film is unable to isolate MSSWs completely, and MSSWs can still be detected that propagate in the $-\vec{H} \times \vec{n}$ direction at the same film surface. However, they are leaked MSSWs that propagate along the $\vec{H} \times \vec{n}$ direction at the opposite surface, and the amplitudes of those leaked waves reduce exponentially as the film gets thicker. Eq. (3) can be used to calculate how large the leaked signal is through the film. This work may lead to the realization of micron-sized microwave isolators, spin-wave diodes (where MSSWs propagate only in one direction), and the study of
MSSWs at an obstacle because the simple reflection of the MSSWs at the obstacle is forbidden.

M.B. thanks Wanjun Jiang and Pedram Khalili Amiri for fruitful discussions, and L.B. acknowledges NSF and an Intel Fellowship for support. This work was supported in part by the DARPA NVL program, NSFC (No. 61131005), the Nanoelectronics Research Initiative (NRI) through the Western Institute of Nanoelectronics (WIN), and the FENA Center.

${ }^{1}$ C. Herring and C. Kittel, Phys. Rev. 81, 869 (1951).

${ }^{2}$ R. W. Damon and J. R. Eshbach, J. Phys. Chem. Solids 19, 308 (1961).

${ }^{3}$ R. E. Camley, T. S. Rahman, and D. L. Mills, Phys. Rev. B 23, 1226 (1981).

${ }^{4}$ N. S. Almeida and D. L. Mills, Phys. Rev. B 53, 12232 (1996).

${ }^{5}$ T. J. Silva, C. S. Lee, T. M. Crawford, and C. T. Rogers, J. Appl. Phys. 85, 7849 (1999).

${ }^{6}$ M. Covington, T. M. Crawford, and G. J. Parker, Phys. Rev. Lett. 89, 237202 (2002).

${ }^{7}$ V. Vlaminck and M. Bailleul, Science 322, 410 (2008).

${ }^{8}$ Y. Kajiwara, K. Harii, S. Takahashi, J. Ohe, K. Uchida, M. Mizuguchi, H. Umezawa, H. Kawai, K. Ando, K. Takanashi, S. Maekawa, and E. Saitoh, Nature 464, 262 (2010).

${ }^{9}$ L. Bai, M. Kohda, and J. Nitta, Appl. Phys. Lett. 98, 172508 (2011).

${ }^{10}$ X. Xing, Y. Yu, S. Li, and X. Huang, Sci. Rep. 3, 2958 (2013).

${ }^{11}$ S. Urazhdin, V. E. Demidov, H. Ulrichs, T. Kendziorczyk, T. Kuhn, J. Leuthold, G. Wilde, and S. O. Demokritov, Nat. Nanotechnol. 9, 509 (2014).

${ }^{12}$ B. Lax and K. J. Button, J. Appl. Phys. 26, 1184 (1955).

${ }^{13}$ P. K. Amiri, B. Rejaei, M. Vroubel, and Y. Zhuang, Appl. Phys. Lett. 91, 062502 (2007).

${ }^{14}$ T. Schneider, A. A. Serga, T. Neumann, and B. Hillebrands, Phys. Rev. B 77, 214411 (2008).

${ }^{15}$ P. K. Amiri, B. Rejaei, Y. Zhuang, M. Vroubel, D. W. Lee, and S. X. Wang, IEEE Trans. Magn. 45, 4215 (2009).

${ }^{16}$ K. Sekiguchi, K. Yamada, S. M. Seo, K. J. Lee, D. Chiba, K. Kobayashi, and T. Ono, Appl. Phys. Lett. 97, 022508 (2010).

${ }^{17}$ A. Khitun and K. L. Wang, Superlattices Microstruct. 38, 184 (2005).

${ }^{18}$ M. P. Kostylev, A. A. Serga, T. Schneider, B. Leven, and B. Hillebrands, Appl. Phys. Lett. 87, 153501 (2005).

${ }^{19}$ T. Schneider, A. A. Serga, B. Leven, B. Hillebrands, R. L. Stamps, and M. P. Kostylev, Appl. Phys. Lett. 92, 022505 (2008).

${ }^{20}$ S. O. Demokritov and B. Hillebrands, in Spin Dynamics in Confined Magnetic Structures I, Topics in Applied Physics Vol. 83, edited by B. Hillebrands and K. Ounadjela (Springer Berlin Heidelberg, 2002), pp. 65-93.

${ }^{21}$ M. Bao, K. Wong, A. Khitun, J. Y. Lee, Z. Hao, K. L. Wang, D. W. Lee, and S. X. Wang, EPL 84, 27009 (2008). 